\title{
NFC Technology Payment System for Android Operation System
}

\author{
Taweechai Anguranak \\ Suan Sunandha Rajabhat University, Dusit Province, Thailand. \\ * Corresponding author. Email: taweechai.an@ssru.ac.th \\ Manuscript submitted July 19, 2015; accepted September 17, 2015. \\ doi: $10.17706 /$ jsw.10.11.1265-1273
}

\begin{abstract}
This purpose of this research was the usage of NFC Technology which installed on the smart phones under the android operation system. It was used for paying bills which used at computer labs of computer science and technology program like printing charges: A4 color, and black and white. By using the android smart phones that supported with NFC Technology, students could touch onto the ACR122U desktop reader which connected to computers for send students' ID information to an application for payment. As the result of system test by using the smart phones, Samsung Galaxy Nexus could pay the service charges at computer's labs. The assessment of efficiency was conducted by using a developed system from the data of questionnaires by asking 15 users. The total results show that the average of users equals 4.20 , and the standard deviation equals 0.44 . This developed system showed a very good quality, and in the result available to use.
\end{abstract}

Key words: Android, near field communication , NFC technology, mobile payment for android.

\section{Introduction}

The computer labs are labs that were able to be used by computer and science major students. The students could use the computers without any payment, but there would be some payments like printing charges, scanner, CD/DVD transcripts as example .

$\begin{array}{lr}\text { Printing } & \\ \text { A4 Black and white } & 1 \text { bath/page } \\ \text { A3 Black and white } & 2 \text { bath/page } \\ \text { A4 color } & 7 \text { bath } / \text { page } \\ \text { A3 color } & 14 \text { bath } / \text { page } \\ \text { Scan } & \\ \text { A4 } & 0.50 \mathrm{bath} / \mathrm{scan} \\ \text { Copy } & \\ \text { CD plus media } & 10 \mathrm{bath} / \mathrm{cd} \\ \text { DVD plus media } & 15 \mathrm{bath} / \mathrm{dvd}\end{array}$

Nowadays, most students have their own smart phones that support with NFC Technology. So, the purpose of the research is to take these enabled NFC Technology smart phones to pay bills which used at computer labs.

NFC Technology is the technology which its basic was from Radio Frequency Identification (RFID) that used the communication system of short-range wireless technologies within only 4-10 centimeters of 
distance. It was developed by Sony and NXP by using $13.56 \mathrm{MHz}$ [1] of frequency wave under the certification of ISO 14443 [2] (Philips Mifare and Sony's FeliCa) [3] which set by NFC Forum that received and sent data at 106 - 424 Kbits of speed per second [4] as the universal standard of ISO/IEC18092:2004 [5]. It's the identifiable and safe technology that has been popular in nowadays. We could see that the smart phones and new mobile phones might come along with NFC Technology. For android system smart phones, this technology may come along with Android 4.0 Ice Cream Sandwich or more, there are some usage qualities of the enabled NFC smart phones.

It works as RDIF Tag which its style of process is like Contactless Smart Card such as E-Card. Most of working process usually goes with financial applications like: fast food service, ticket vending machine, and especially for low amount of payment that could be more accommodative to consumers, and it could also add the value proposition for business. The consumers could bring their enabled NFC smart phones to touch onto the NFC Reader Machine which placed at payment point. So, it could pay bills instead of paying by cash or E-card.

It works as NFC Reader Machine like Contactless Smart Card Reader/Writer Machine such as: reading data from attached tag in Smart Poster. It's kind of applied work process is to encourage the marketing by giving smart phone users free discount coupons. Communication as Peer to Peer, this mode may synchronize data with NFC device like: songs, name card, photos, etc.

\section{Relate work}

\subsection{Android}

Android [6] is the operation system which based on the operation system work, Linux, for portable devices like: smart phones and tablets. It was developed by Any Rubin who set up Android, Inc in 2003. Nowadays, android has been developed by Google in user interface's part which based on direct manipulation. NFC Technology has been put in the smart phones in everyday's life. NFC technology for android operation system version 4.0 or higher, there will be NFC named, "Android Beam", like: Samsumg Galaxy Nexus.

\subsection{NFC Operating Modes}

NFC Operating Modes [7] could be separated into 3 modes: there are reader/writer, peer-to-peer, and card emulation as the Fig. 1.

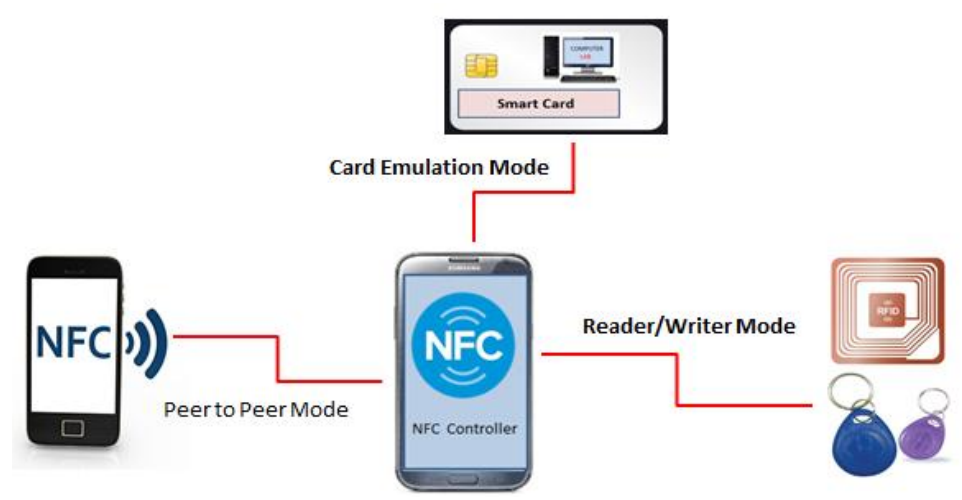

Fig. 1. NFC operating modes.

\subsubsection{Reader/Writer mode}

This mode, NFC device may work as contactless smart card reader/writer machine, and it's also called RFID Tag. This following mode is related to ISO/IEC 14443 standard (ISO 14443-A/B or FeLiCa) such as: 
reader mode, it's used for reading smart poster application on NFC device. Reader/Writer mode [8] is used for the process of reading and writing into wanted tags. And the smart phones which support with NFC in reading and writing mode with NFC tags aim to read and write the data into the wanted tags.

\subsubsection{Peer-to-peer mode}

This mode, two NFC enabled mobile devices will communicate and exchange data together, the data may be sent through bidirectional half duplex channel. So, when the one is sending data, another one will receive and start to transmit data after the first one finishes as the applications of Peer-to-Peer Mode under the standard of ISO 18092 such as: exchanging money transfer and social networking.

\subsubsection{Card emulation mode}

This mode may work as contactless smart card that means NFC enabled mobile service might work as contactless smart card, and there will be communication in receiving and sending data based on ISO/IEC standard (ISO/IEC 14443 Type A and Type B, and FeliCa) The usage of only one device could be kept as various kinds of smart card such as: BTS ticket issuing, E-Purse card of Thai Smart Card which used at 7-11, credit card, debit card, loyalty card, transport cards, and identity or access cards. There are some applied usages of card emulation mode like: mobile payments, loyalty programs, e-ticketing and access control.

\subsection{Mobile Payment}

Mobile Payment[9] is a kind of transactions such as: money transfer, goods payment, and other internet services instead of paying by cheque or credit card. There are 4 models of mobile payment as these followings.

\subsubsection{Paying mobile bills}

Paying mobile bills is the way to pay bills via SMS (short message service). The customers could pay the bills and services via SMS or USSD (Unstructured Supplementary Service Data) by typing the keyword and unique number, and sending messages to the number as customers informed. For the payment of sending messages, bills and services of device system, prepaid, the systems may immediately deduct from balance. For monthly systems, there will be bills that customers need to pay sending as a letter and MMS. Most of these kinds of payment are usually goods and services that sent to the customers via MMS such as: downloads, songs, ringtones, and pictures.

\subsubsection{Direct mobile billing}

It's one of the ways to pay bills. The customers could pay them by mobile billing through e-commerce such as: online gaming which allows the customers to pay bills, and services mobile phone. During the payment, users must inform their telephone number and OTP (one time password). When the verification has done, the customer's mobile account will be activated for purchasing. At these moments, the direct mobile billing has become popular and famous in Asia continent.

\subsubsection{WAP (Mobile web payments)}

The customers could use sites to show or download applications to install on their devices for paying bills through internet which is the basic technology and supports with wireless application protocol. This kind of payment, the customers could pay bills by credit card, debit card, bank account, and e-money.

\subsubsection{Mobile billings by NFC technology (Near field communication)}

Most of smart phone users usually use NFC Technology to pay bills for purchasing goods at stores and taking transportation, and they just bring their NFC device to touch onto the reader machine (payment machine) for transaction. Most of this way of mobile billing has no authentication but some of transactions 
must be checked by PIN. The payment will be deducted from prepaid account or directly billed through bank account or devices before the transaction has done. There are some examples of services such as: bus and sky-train payment, store payment, and restaurant payment.

\section{Architecture Design of System}

As the second picture, payment with NFC technology has been developed: it's composed by applications on android system of smart phone which belongs to users or members, and application on PC of computer lab's officers. The users or members (No.1) could fill in student's ID by their device for sending it through SNEP [10] (simple NDEF exchange protocol), Android Beam (send NDEF messages via Android Beam)[11], [12] to Java application which connects to ARC122U[13] (No.2). This application may sent student's ID to web application (No.3) that connects to database (No.4) for process as the functions that users choose in web application.

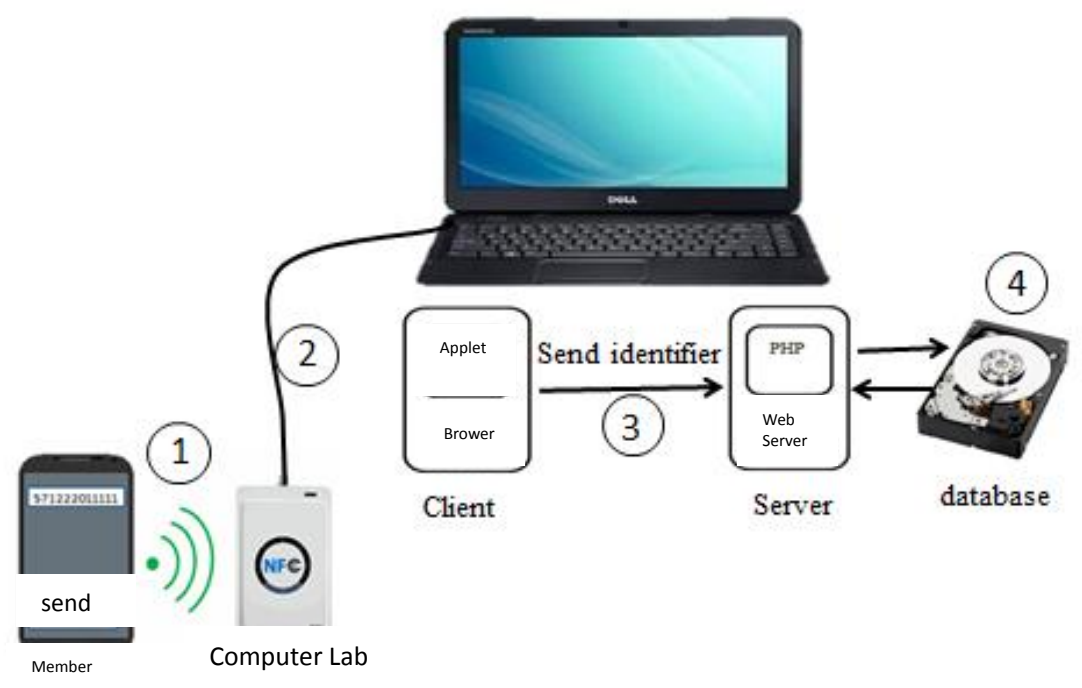

Fig. 2. Architecture design of payment system of NFC technology.

For example, as the second picture, code number 3 which from browse client sends information to web server by GET Method for setting login.php, as this followings.

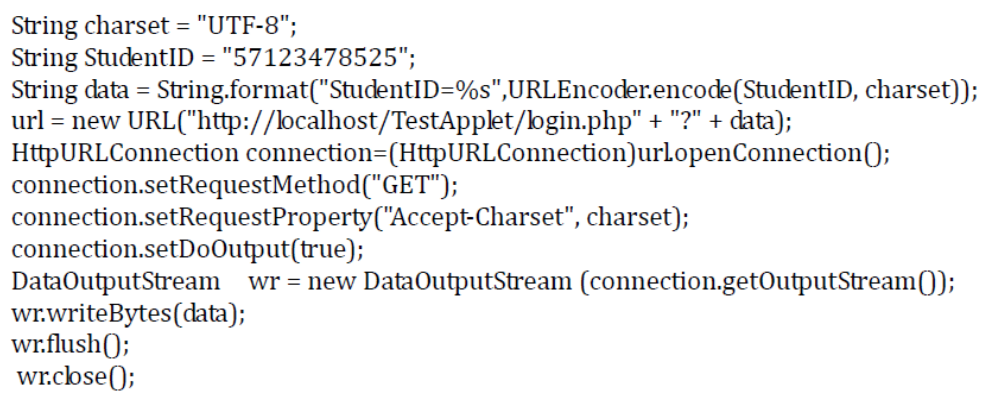

\section{Database Design}

Database design of payment with NFC technology system can be separated into 4 relations: there are member, service, service detail, and service charge as the Fig 3.

Entity - Relationship Diagram 


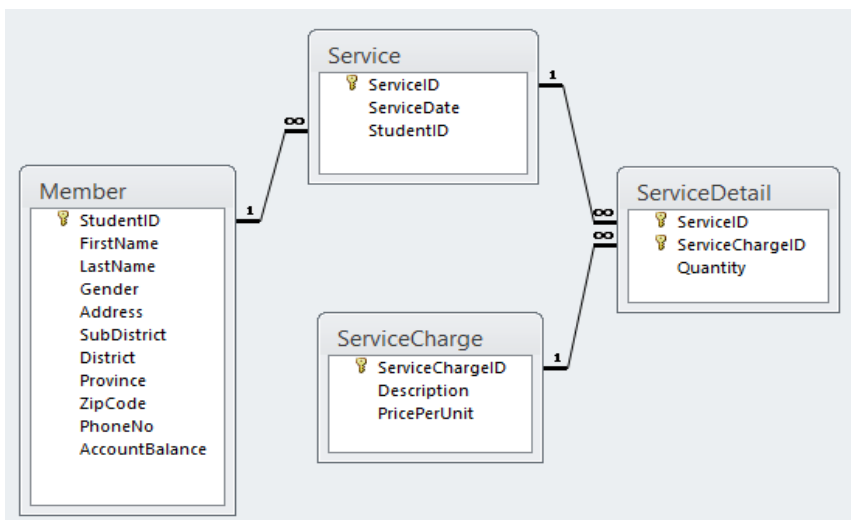

Fig. 3. The charts that shows the relationships of data system of payment with NFC technology.
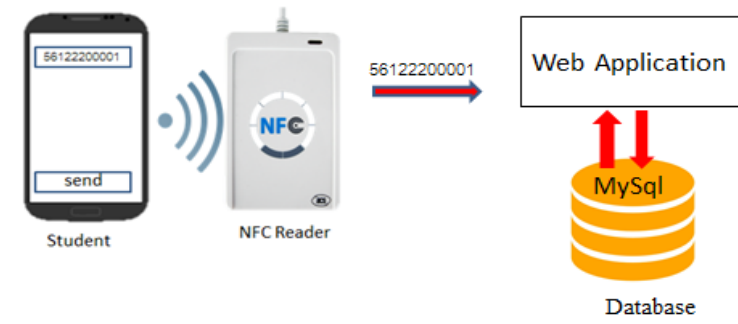

Fig. 4. The process of payment system by using smartphone.

\section{System Development}

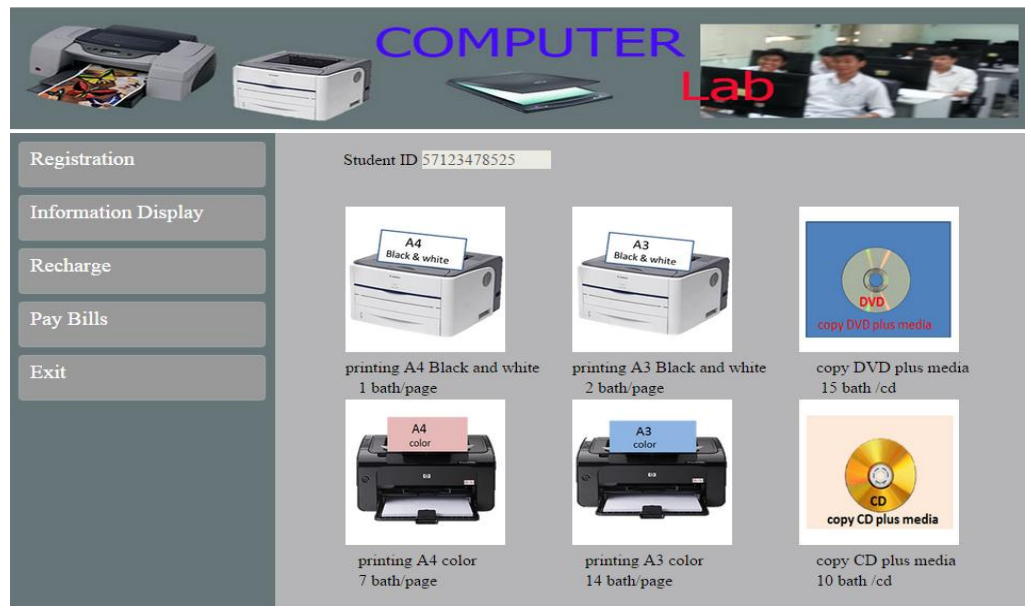

Fig. 5. Main webpage.

The system development of service charges payment with NFC devices is composed of two parts program. The first section application would be installed on Android 4.2 system's smart phone which supported to NFC technology. The second one will be installed on the computer's desktop as a program that connects to the ACR122U desktop reader, and web server that develops payment system by PHP language. So, the students using NFC technology smart phone could send their ID to the application that connects to the ACR122U desktop reader. After that, the application will send the students' ID to the webpage's payment application as the Fig. 4. The payment system of application on website has various lists on the main webpage. There are registration, information display, recharge, and payment as the Fig. 5.

\subsubsection{Registration}


The Students or members have to register before accessing services at computer labs. Students using NFC smart phone could send information and students' ID to the application connected to the ACR122U desktop reader for registering. Then, officer's computer will fill the student's data as Fig. 6.

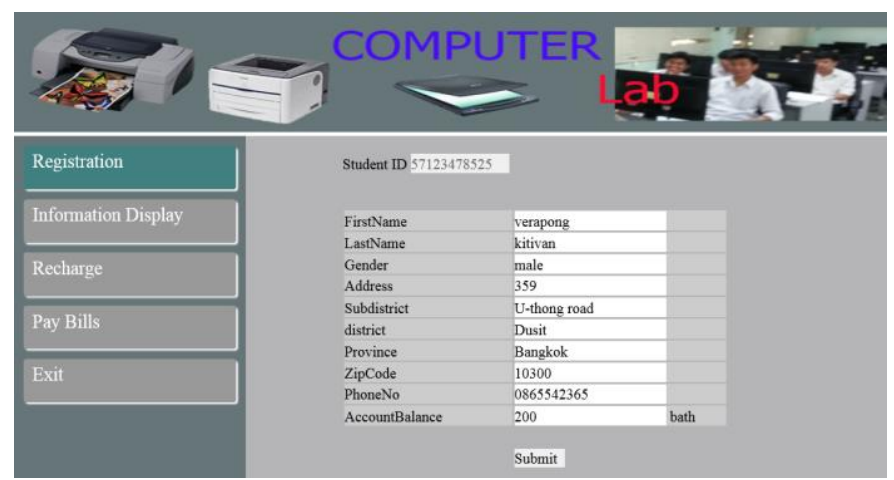

Fig. 6. Registration.

\subsubsection{Information display}

The Students could check their information from officer's computer by using NFC smartphone to send the information and students' ID to the application that connected to reader machine for displaying students' data and balances as the Fig. 7 .

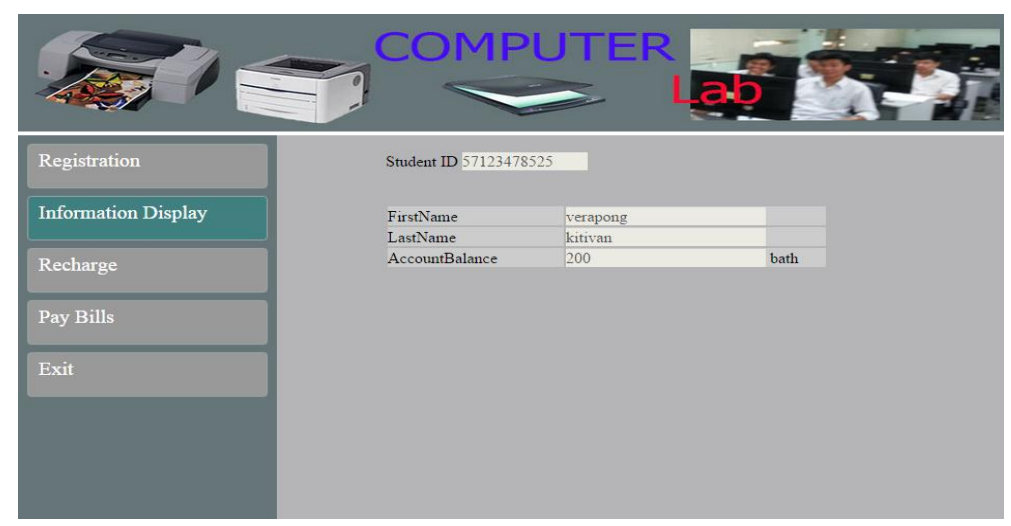

Fig. 7. The display of student's data and balances.

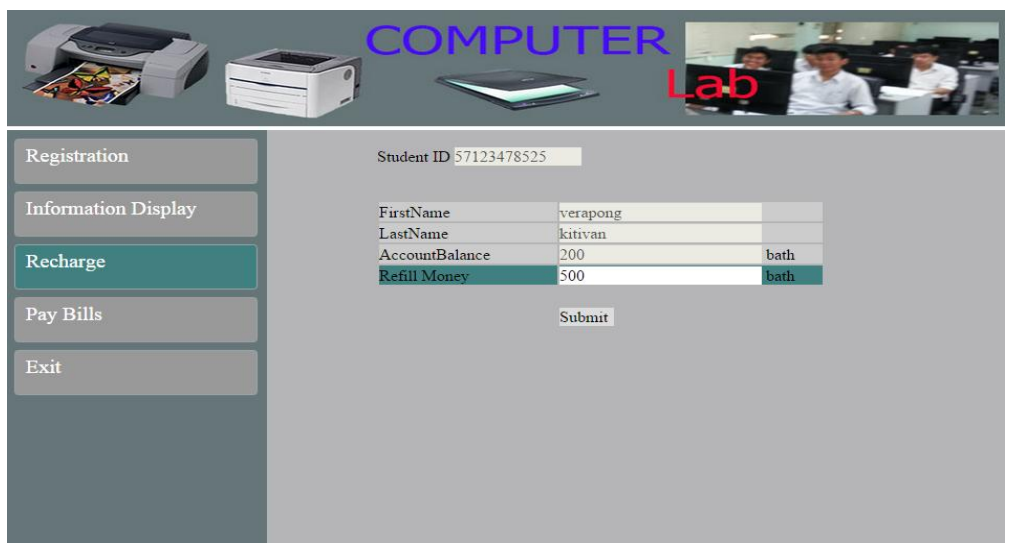

Fig. 8. Recharge.

\subsubsection{Recharge}

The Students could refill money from officer's computer by using NFC smart phones to send student's ID 
to the application connected to the ACR122U desktop reader. And, on the application's webpage, the officer's computer would fill the money's amount that student need to fill, and save it as the Fig 8.

\subsubsection{Pay bills}

The students could pay bills and services at the officer's computer by using android smart phone to send data and student's ID. Then, the officer will save the lists that needed to be paid and deduct the balance as the Fig. 9.

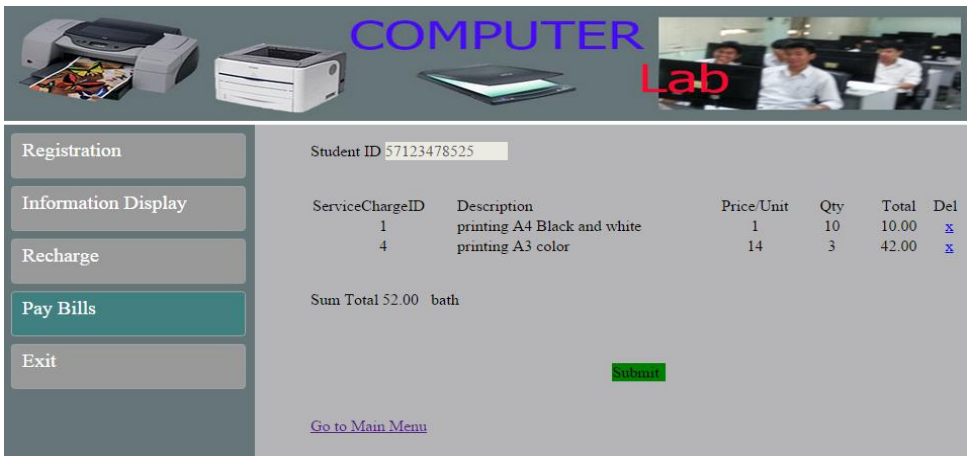

Fig. 9. Pay bills.

Table1. The Criterion of EVALUATION Marking

\begin{tabular}{cl}
\hline \hline Scale of Evaluation & Signification \\
\hline 5 & Strongly Agree \\
\hline 4 & Agree \\
\hline 3 & Undecided \\
\hline 2 & Disagree \\
\hline 1 & Strongly Disagree \\
\hline \hline
\end{tabular}

Table 2. The Interpretational Criterion of Data By Considering the Average

\begin{tabular}{cl}
\hline \hline Scale of Evaluation & Signification \\
\hline $4.21-5.00$ & Excellent \\
\hline $3.41-4.20$ & Very Good \\
\hline $2.61-3.40$ & Average \\
\hline $1.81-2.60$ & Fair \\
\hline $1.00-1.80$ & Poor \\
\hline \hline
\end{tabular}

\section{System Evaluation}

System Evaluation is an acceptance test by users for evaluating the efficiency of a developed system, by splitting 3 parts of system evaluation as follows:

1) Functional Requirement Test

2) Functional Test

3) Usability Test

Evaluation is specified by the standard of quantitative marking, the five ranks sort of rating scale assessment following the step of Likert [14]. There are details to specify the score rank, and the scale of weight in system contentment as shown in Table 1 and Table 2.

\section{Conclusion}


The purpose is to evaluate the developed efficiency of development of NFC Technology Payment System for Android Operation System. The average equals to 4.20 and the standard deviation equals to 0.44 from the group of 15 users. So, we can conclude that this developed system has a very good quality of satisfaction as shown in Table 3.

Table 3. The Result of Evaluation of Satisfaction with the System

\begin{tabular}{lcc}
\hline \hline \multirow{2}{*}{ The Evaluation of the System } & \multicolumn{2}{c}{ The Common Users } \\
\cline { 2 - 3 } & $\bar{x}$ & SD \\
\hline Functional Requirement Test & 4.24 & 0.49 \\
\hline Functional Test & 4.19 & 0.39 \\
\hline Usability Test & 4.17 & 0.44 \\
\hline Total Average & 4.20 & 0.44 \\
\hline \hline
\end{tabular}

\section{Future Work}

From the system demonstration, it shows that needed development to be able to make income-expense account of taking services at computer labs, there should be security system which separated into two sections between officers and students.

\section{Acknowledgements}

This study is supported as part of a project funded by The Institute of Research and Development Suan Sunandha Rajabhat University (www.ssru.ac.th).

\section{References}

[1] Vedat, C. K. 0., \& Busra, O. (2012). Near Field Communication (NFC): From Theory to Practice. Wiley Publishing.

[2] Gerald, M., \& Josef, L.(2008). Managing an NFC ecosystem. Proceedings of the IEEE 7th International Conference on Mobile Business ICMB 2008.

[3] NFC forum technical specifications. Retrieved August 12, 2014, from http://members.nfc-forum.org/ specs/spec_list/

[4] Finkenzeller, K. (2010). RFID Handbook: Fundamentals and Applications in Contactless Smartcards, Radio Frequency Identification and Near-Field Communication. Wiley.

[5] ISO/IEC 18092:2004 Information technology — Telecommunications and information exchange between systems - Near field communication - Interface and protocol (NFCIP-1). Retrieved December 11, 2011, From http://www.iso.org/iso/catalogue_detail.htm?csnumber=38578

[6] Android operating system. Retrieved September 6, 2014, from http://en.wikipedia.org/wiki/ Android_(operating_system)

[7] Nattaphon, S., \& Chate, P. (2014). An membership system for android smartphone with NFC card emulation mode. Proceedings of the NCCIT 2014: The Tenth National Conference on Computing and Information Technology (pp. 908-913). Angsana Laguna Phuket, Thailand.

[8] Vedat, C., Kerem, O., \& Busra, O. (2013). Professional NFC Application DEVELOPMENT for Android. Wrox Press Ltd., Birmingham,UK.

[9] Mobile payment. Retrieved January 20, 2014, from http://en.wikipedia.org/wiki/Mobile_payment

[10] Open source library to facilitate P2P communication over NFC (SNEP). Retrieved February 15, 2014, from http://code.google.com/p/ismb-snep-java/

[11] Open source LIBRARY to facilitate P2P communication over NFC (NPP). Retrieved May 22, 2014, from 
https://code.google.com/p/ismb-npp-java/wiki/NppFromPhoneToACR122

[12] Tom, I., Don, C., \& Brian, J. (2014). Beginning NFC Near Field Communication with Arduino, Android, and Phone Gap. O'Reilly Media: Publisher.

[13] ARC122U USB NFC reader. Retrieved October 10, 2014, from http://www.acs.com.hk/en/products/3/ acr122u-usb-nfc-reader

[14] Likert, R. (1932), A technique for the measurement of attitudes. Archives of Psychology, 140, 1-55.

Taweechai Anguranak received his MSc in computer science from the National Institute of Development Administration, Bangkok, Thailand. He is currently working as an associate professor in the Department of Computer Science, Suan Sunandha Rajabhat University. His research interests include mobile computing, web ontologies and semantic web, database system. 